\title{
MATHEMATICAL MODEL APPLIED TO IMPROVE THE NATURAL LIGHTING DESIGN
}

\author{
B. GARCÍA-FERNÁNDEZ ${ }^{1}$, D. VÁZQUEZ-MOLINI ${ }^{1}$ \& A ÁLVAREZ FERNÁNDEZ-BALBUENA ${ }^{1,2}$ \\ ${ }^{1}$ Department of Optics. Faculty of Optics and Optometry, University Complutense of Madrid, Spain. \\ ${ }^{2}$ Department of Forest and Environmental Engineering and Management, School of Forestry Engineering, \\ Polytechnic University of Madrid, Spain.
}

\begin{abstract}
Daylighting must be considered as the greenest way to illuminate any space and one of the most important architectural design challenges and opportunities. Human life is strongly influenced by natural lighting, and nowadays we have to add to this factor the importance of energy conservation and pollution reduction objectives. The development of hollow light guides offers people the advantages of natural lighting and the benefits of reduced energy consumption systems, providing changes in architectural form of spaces where sunlight does not have direct access. Hollow Cylindrical Prismatic Light Guides (CPLGs) are transparent optical components able to transmit high diameter light beams in daylight buildings applications without relevant losses. The goal of this paper is to show a novel lighting design based on hollow prismatic light pipes, which let daylight to be introduced inside of buildings. An improved mathematical modelling to predict light transmission efficiency based on realistic software simulations and experimental models is presented.
\end{abstract}

Keywords: cylindrical prismatic light guide, prismatic film, prisms defects, transmission efficiency, transmittance model.

\section{INTRODUCTION}

The use of natural light inside buildings provides high quality lighting, reduces energy consumption and increases comfort and productivity. Light pipes can transfer light from a building's roof into the depths of the building, thus the overall wattage of installed lights is reduced, and therefore the consumption of electricity decreases due to lesser use of artificial lighting. Tubular light guides are a complementary product to conventional skylights and windows which can complement the artificial lighting in areas not usually covered by windows and skylights [1].

Prismatic light guides are hollow structures internally covered with prismatic sheet, they offer a technical alternative as a light transport in the tubular guidance systems as the usual hollow light guides use composed of aluminium. The prismatic light guide leads light beams by Total Internal Reflection (TIR) and provides light transmission with high efficiency and homogeneous light distribution in buildings with minimal colour shifts [2].

The prismatic film has one flat surface and the other one consists of a textured surface with extruded prisms. The material can work as reflective or transmissive depending upon the angle at which a light ray strikes the surface of the film. In CPLG, light rays are propagated by TIR when the input light is incident under the angle accepted for flux transmission [3]. Light that doesn't fulfil this requirement is partially extracted out of the guide. Figure 1 depicts a light 
Cylindrical Prismatic Hollow Light Guide

a)
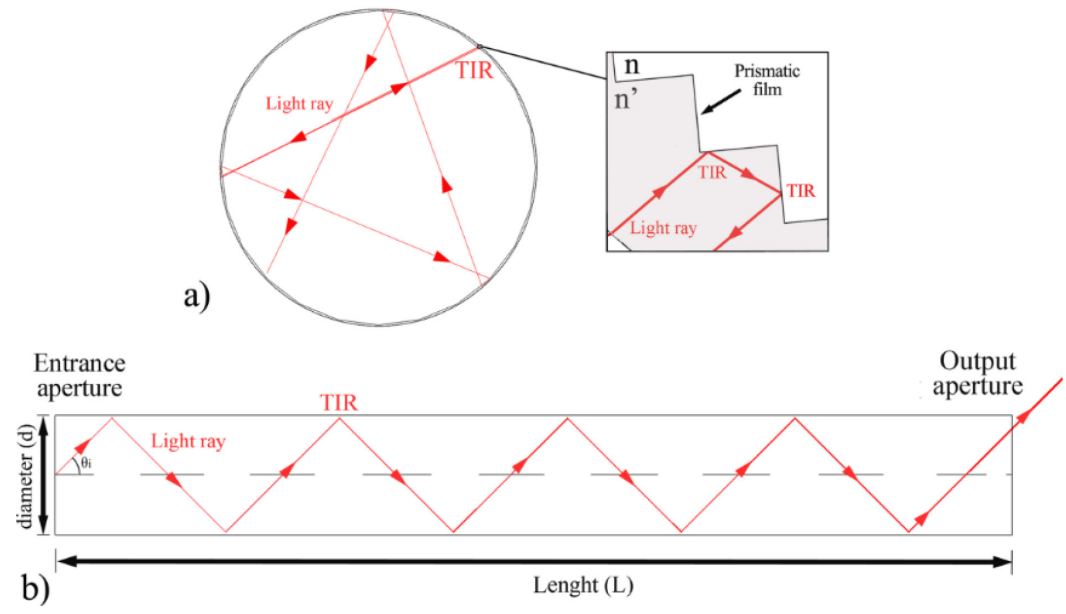

Figure 1: Sketch of hollow CPLG performance showing an arbitrary ray travelled down the hollow prismatic guide by multiple TIR. (a) Transversal view. (b) Longitudinal view with a detail of TIR in the microprismatic structure.

ray transmitted through the CPLG by TIR. The transverse motion with a detail of TIR in prismatic structure of optical film is shown in Fig. 1a. The schematic illustration shows in lateral view (Fig. 1b), an arbitrary ray transmitted by the guide undergoing by multiple TIR. The dimensions used to characterize the hollow guides are given: length $(L)$, diameter $(d)$ and aspect ratio $(\rho)$ defined as length to diameter $(\rho=L / d)$.

The shape and size of the pipe can vary depending on the desired optical function and the object to be illuminated. Moreover, the amount of light extracted in a position of the guide can be controlled by making changes in the prisms [4].

The use of prismatic thin film is valuable because it allows reducing the optical path length in dielectric media. Consequently, the minimal light absorption losses due to bulk absorptivity of the material usually make the film reach high transmittance and thus, it is possible to obtain a high transmission in prismatic guides used in light distribution systems with minimal losses. Despite the fact that manufacturing process in actual technologies makes possible the development of optimal prismatic film, some geometric parameters as fabrication defects can affect the light output efficiency [5-7]. Nowadays, thanks to the current technological tools accurate mathematical models can be developed to predict the performance of prismatic light guides, avoiding previous technological limitations.

In this paper, by ray tracing and subsequent analysis is possible to evaluate the factors that can affect the prismatic guide's efficiency and thus accurately predict their influence. The guide transmittance $(T g)$ used to characterize the flux of light transmitted within the interior of the guide, is defined as $T g=\phi_{d} / \phi_{i}$ where $\phi_{d}$ is the flux intercepted by the screen detected at different positions of the source and $\phi_{i}$ is the flux at a given input location.

The angular characteristics of the lighting system a factor that determines the efficiency guided as will be demonstrated experimentally, the incident light ray location produces changes in the beam path that suggests the need to quantify the efficiency depending on the size of the light source area. 


\section{RAY TRACING SIMULATIONS}

Tridimensional simulations were carried out in a non-sequential optical ray tracing software, TracePro 7.4 [8]. Firstly, we check the influence of the light beam divergence angle to specify the angular acceptance range for optimal transmission of light by realistic simulations. Secondly, an analysis of the influence of radius in peaks allows us to quantify the losses due to surface errors along with topographical surface analysis and experimental simulations. Finally, an irradiance analysis will be shown by varying the diameter of the source.

In simulations, a realistic prismatic sheet with constructive prismatic parameters whose base is $356 \mu \mathrm{m}$ wide and its height is $178 \mu \mathrm{m}$ [9] is designed to model several prismatic hollow light guides (Fig. 2). The efficiency was evaluated along the interior of the guides at different equidistant distances.

In order to generate a suitable 3D model for a raytracing evaluation some approximations in the geometry profile were used. We consider the corners in cross section as a radius of curvature $r$ as (shown in Fig. 3). This approach is made taking to account the relation of the circle with the radii linearly proportional to trigonometric function: $x=2 r \operatorname{sen}(\pi / 2)$, where $x$ is considered the chord length, $\alpha$ is the angle subtended at the centre by the chord $\left(90^{\circ}\right)$ and $r$ is the radius set by the enrolled circle.

The influence of defects in prism corners is shown by the design of different 3D guides with several corner radii. In addition, as an ideal reference, the perfect prism $(r=0)$ was evaluated. The radius of curvature has been set to be $0,3,6$ and $9 \mu \mathrm{m}$. The wavelength was set at $590 \mathrm{~nm}$ in calculations. The refractive index material of the guide is considered 1.59



Figure 2: Perspective view of raytrace simulation model with a detail of the cylindrical prismatic structure.

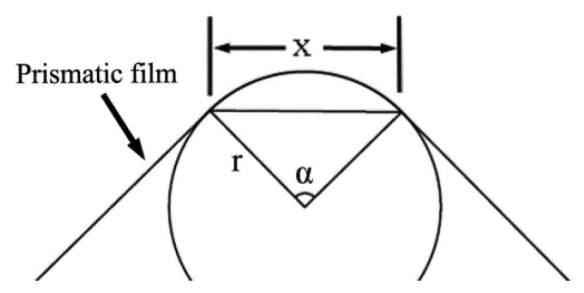

Figure 3: Geometrical description in cross-section to determine the radius of curvature used in the transmittance model $\left(\alpha=90^{\circ}\right)$. 
determined by using a polycarbonate polymer. Coefficient of absorptance in the prismatic material has been analysed over the range of 0 to $0.1 \mathrm{~mm}^{-1}$.

\subsection{Influence of light beam divergence angle}

The angular distribution of the input light is a critical parameter that modifies the flux transmission with regard to distance down the prismatic guide. In this section, the influence of the input light beam angle is studied to specify an angular acceptance range for optimal transmission of light. For this analysis, the entrance aperture is considered uniformly illuminated with a clipped lambertian emission source of $8 \mathrm{~mm}$ diameter spot size with a random distribution, a detector is situated at the end of the CPLG in order to achieve the light flux. Figure 4 shows the decrease of efficiency in the perfect prismatic model $(r=0)$ with regard to 3,6 and $9 \mu \mathrm{m}$ radius in corners with an aspect ratio of 30 . The transmission efficiency decreases with increasing the angle of the light cone. The decrease in slope is emphasized when the angle analysed is out of the range for which the prismatic film provides higher efficiency [10]. Beyond light cone's semi-angle of $30^{\circ}$ the transmission curve suffers a change in the slope, this is due to the fading of the TIR refraction. In Fig. 5 is marked with a vertical line the angle where there are a change in slope (aprox. $30^{\circ}$ ). The results indicate that the change of transmission efficiency in ideal prism with this configuration reproduced is minimal; being of the order of $4.9 \%$ in the $30^{\circ}$ semi-angle with an aspect ratio of 30 . The transmission through the guides with defects in corners suffers a notable decay even for $3 \mu \mathrm{m}$ radius evaluated, in this specific case, the efficiency decreases about $29.2 \%$. Examination of the data shows that, approximately, the maximum values attained correspond to $30^{\circ}$. Therefore, the light source of the optical measurement method has been carried out by with the approximately maximum acceptance angle attained, a clipped Lambertian source with a semi-angle cone of $30^{\circ}$ at the input which integrate rays over all suitable directions.



Figure 4: Transmission efficiency affected by the maximum incidence angle of the light source with an aspect ratio of 30 for different roundness values in corners: $0,3,6$ and $9 \mu \mathrm{m}$. 


\subsection{Impact of corners defects in light performance}

We present an analysis related to the corners defects. In the simulations, the entrance aperture is considered uniformly illuminated with a clipped Lambertian emission source for which the prismatic film provides higher efficiency. The light source is an emitter of $8 \mathrm{~mm}$ diameter spot size with a random distribution, which emits in a semi-angle of $30^{\circ}$, CPLG were measured with aspect ratios from 0 to 49 . The dimensions used to characterize the hollow guides are given as aspect ratio $(\rho)$.

Table 1 shows transmission efficiency between cylindrical prismatic guides (absorption coefficient of $1 \cdot 10^{-3} \mathrm{~mm}^{-1}$ ) which have different curved regions showed in percentage which depend on the aspect ratio of the light guide $(\rho)$. Table 1 represents efficiency transmitted for various radius peaks versus several aspect ratios of cylindrical prismatic guides with curved regions with radios of 3,6 and $9 \mu \mathrm{m}$.

Table 1 shows that the decrease in efficiency obtained for CPLGs is highly related to the corners defects, in this case, approximated by a radii. Their influence is a critical parameter mainly when the aspect ratio of light guides increases. In perfect prism, there are losses mainly due to the bulk absorption coefficient of the material. The percentage of global bulk absorption obtained in an ideal light guide with perfect prism and an aspect ratio of 30 is $2.13 \%$.

\subsection{Influence of light source area in transmission efficiency}

A centred light emitter compound of a circular random grid source emitting in a semi-angle of $30^{\circ}$ is incorporated at the beginning of the guide. The influence of the light source area in

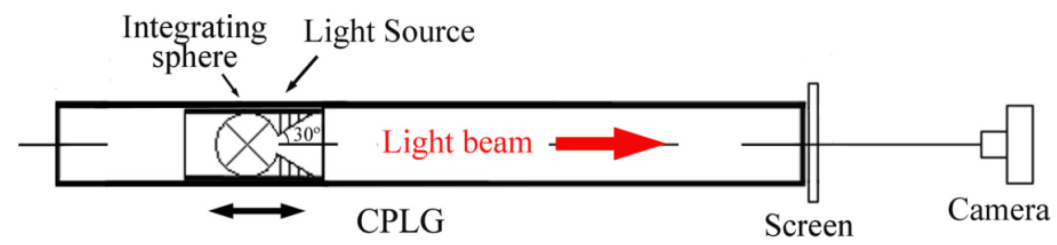

Figure 5: Schematic lateral view of the experimental set-up. The light source is displaced through the inner guide while a camera records the output flux for different lengths.

Table 1: Transmission efficiency for various radius peaks versus several aspect ratios of cylindrical prismatic guides with perfect right angle and peak radios of 3, 6 and $9 \mu \mathrm{m}$.

\begin{tabular}{lrrrrr}
\hline \multirow{2}{*}{ Aspect ratio $(\rho)$} & \multicolumn{5}{c}{ Transmission efficiency (\%) } \\
\cline { 2 - 6 } & 10 & 20 & 30 & 40 & 50 \\
\hline Perfect right angle & 98.95 & 98.33 & 97.87 & 97.20 & 96.48 \\
Peak radius $3 \mu \mathrm{m}$ & 91.62 & 84.65 & 78.64 & 72.28 & 66.63 \\
Peak radius $6 \mu \mathrm{m}$ & 84.45 & 72.44 & 61.87 & 53.44 & 46.15 \\
Peak radius $9 \mu \mathrm{m}$ & 78.56 & 63.20 & 49.97 & 40.64 & 32.96 \\
\hline
\end{tabular}


transmission efficiency was determined using five different diameters. The light sources were scaled in area from 0.01 to 1 (normalized to the maximum value area of the entrance aperture). Figure 6 shows the variation in transmission efficiency for several aspect ratios with $3 \mu \mathrm{m}$ of radii in prism corner and without absorption loss record.

The size of the light source influences the light distribution in the central area of the guide.

By changing the circulating path length, the beam energy varies. The size of the light source produces changes in efficiency because the location of the incident ray light determines the number of reflections inside the guide and therefore the amount of light escaping to the outside due to the prismatic defects.

Transmission efficiency decreases when the light source area increases. For example, in the aspect ratio of 30 , there is a decrease of $5.81 \%$.

\section{EXPERIMENT}

In this section, we present an experimental evaluation of the transmission efficiency and flux distribution in a CPLG. The prismatic guide is a cylindrical guide internally covered with a prismatic film whose outer face is composed of $90^{\circ}$ microprismatic structure longitudinal to the axis of the guide. The light guide has a diameter of $96 \mathrm{~mm}$ and the total length is $3 \mathrm{~m}$. The transmission efficiency of the light guide prototype, for several aspect ratios was experimentally measured using a calibrated USB CMOS Monochrome Camera (DMK 72BUC02, Imaging Source). The light source used is displaced at intervals of 50 millimetres through the guide inserted in a cylindrical device (Fig. 5) to take measurements with different aspect ratios. To ensure Lambertian emission, one LED is positioned on the inner surface of an integrating sphere characterized by a Lambertian reflectance. The cylindrical device that ensures the position of the sphere has three circular diaphragms located coaxially with different diameters to determine the angle cone of the emitting source. An aperture of $8 \mathrm{~mm}$ was used which restrict the output emission from the integrating sphere to accomplish the photometric Lambertian distribution with the $30^{\circ}$ semi-angle cone. The source emitter is an LED Luxeon LXHL-PL01 with the peak emission wavelength of $590 \mathrm{~nm}$, this value is in agreement with the wavelength used in raytracing simulations. The radiant flux was measured by means of a

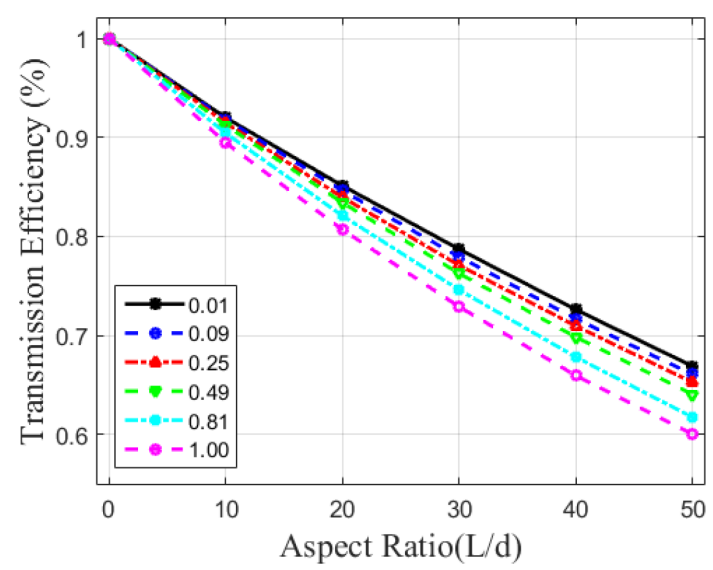

Figure 6: Transmission efficiency affected by the size of the light source (legend scaled in area from $0.01-1$ ). 
camera through which it is obtained the irradiance maps by a Lambertian screen fixed at one end of the guide.

The results show an exponential decrease in the flux of transmitted light through the guide.

Figure 7 shows the radiant flux collected by the screen mapped in extreme positions of the source, the maps has been normalized to the maximum flux value of the zero aspect ratio. Figure 7a shows the flux distribution at the input $(\rho=0)$, it matches the flux emitted by the source. The flux distribution at the output $(\rho=30)$ is concentrated in the central region (Fig. 7b).

Table 2 shows the transmission efficiency as a function of the aspect ratio down the guide.

\section{SEMIEMPIRICAL TRANSMITANCE MODEL FOR HOLLOW PRISMATIC LIGHT GUIDES, CHECKUP EVALUATION}

The simple expression for transmission efficiency assumes the model of light propagation in CPLG using an exponential decay contained several independent exponential terms related to losses. The proposed semiempirical model for prismatic light guide transmittance, takes into account corner defects, absorption and residual losses for different aspect ratios.

Thus, the guide transmittance can be expressed by a mathematical function as:

$$
T_{g}(r, \mu, \rho)=e^{-k 1 r \rho} e^{-k 2 \mu \rho} e^{-k 3 \rho},
$$

where $r$ is the corner radius ( $\mathrm{m}$ ) of the prismatic structure, $\mu$ is the absorption coefficient of prismatic film $\left(\mathrm{m}^{-1}\right)$ and $\rho$ is the aspect ratio of the light guide. $k_{1}, k_{2}$ and $k_{3}$ are considered fitting parameters.

The first term evaluates changes induced in light transmission by defects in prism corners. The second term of the model denotes the influence of absortance. The third term includes several parameters inducing residual losses like scratches, cracks, powder, material inhomogeneity, film junctions, cylinder covering, etc... The first and the second exponential terms
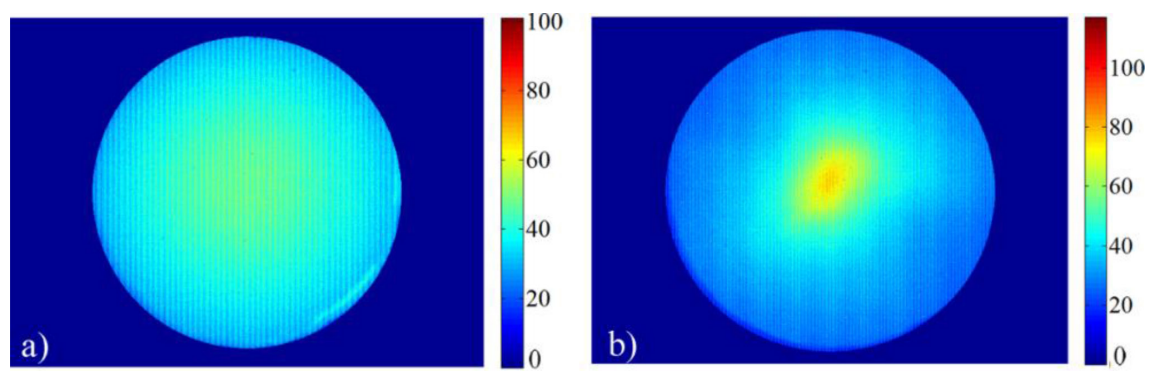

Figure 7: Irradiance map obtained at the input (a) and output (b) of the prismatic guide in experimental assembly $(\rho=30)$.

Table 2: Transmission efficiency in experimental assembly.

\begin{tabular}{lccccccc}
\hline & \multicolumn{7}{c}{ Transmission efficiency (\%) } \\
\cline { 2 - 8 } Aspect Ratio $(\rho)$ & 0 & 5 & 10 & 15 & 20 & 25 & 30 \\
Experimental model & 100 & 95.36 & 90.95 & 88.26 & 82.51 & 79.29 & 73.79 \\
\hline
\end{tabular}


had been empirically calibrated with software simulations. The third additional term incorporates residual losses obtained from the experimental data.

The size of the light source entering the prismatic guide influences the efficiency as seen above. Therefore, in eqn (1) can be added an additional exponential term, which denotes the influence of the size source $A$ :

$$
T_{g}(A, \rho)=e^{-k_{4} A \rho}
$$

The complete equation will be:

$$
T_{g}(r, \mu, A, \rho)=e^{-\rho(k 1 r+k 2 \mu+k 3+k 4 A)},
$$

Eqn (3) is checked for a CPLG illuminated by an acceptance semi-angle $\sim 30^{\circ}$, which approximately maintains the maximum acceptance angle determined by the refractive index of the prismatic film.

The simplex optimization method [11] is applied to obtain the parameters $\mathrm{k}_{1}, \mathrm{k}_{2}$ and $\mathrm{k}_{4}$ of simulations data. Third term $k_{3}$ was obtained from experimental data.

The final adjustment parameters are identified after the optimization procedure as $k_{1}=2.6 \cdot 10^{3} \mathrm{~m}^{-1}, k_{2}=5.8 \cdot 10^{3} \mathrm{~m}, k_{3}=1.3 \cdot 10^{-3}$ and $k_{4}=2.55 \cdot 10^{3} \mathrm{~m}^{-2}$.

In Figure 8, we show the higher agreement between the results obtained of the model proposal taking to account the complete solution of eqn (3) (solid line) comparing with experimental measurements (marked solid line) and simulation data (marked dashed line) of realistic guides for radii in prisms corner of $3 \mu \mathrm{m}$.

The model is able to account defects in prism corners, absorptance, residual losses and light source area. We, therefore, conclude that the decrease in efficiency for CPLG is highly related to the radius in the corners. There is minimal the influence of the other parameters studied.

\section{APPLIED PRISMATIC LIGHT GUIDE SYSTEM}

In this section, a new lighting performance applicable to buildings is presented which include an optimal hollow prismatic light guide system for light transmittance using the sunlight as a

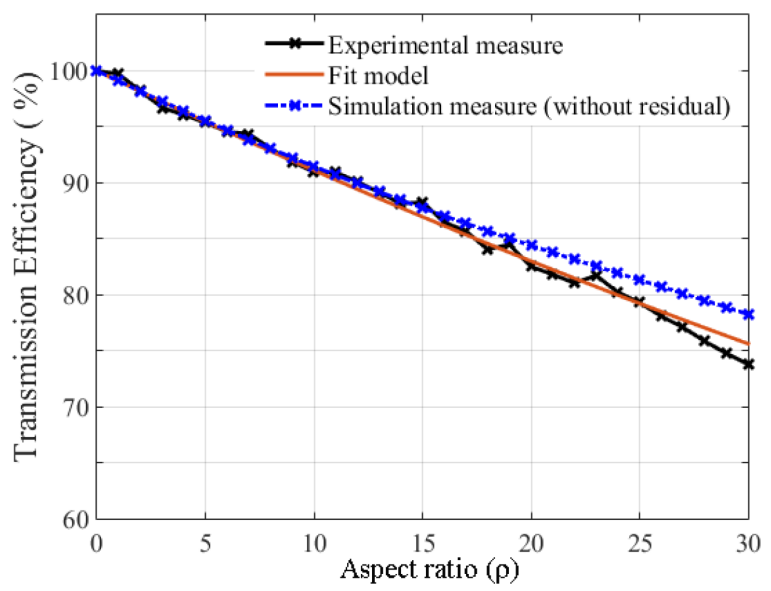

Figure 8: The proposed semiempirical model's fit for prismatic light guide transmittance, experimental measurements and data obtained of realistic simulations for different aspect ratios. 
lighting source. The prismatic lighting system is susceptible of incorporating numerous hollow light guides with several sizes, which can be conformer depending upon the environment and the requirements of use. The system presented is compounded of two cylindrical prismatic light guides (CPLGs) with aspect ratios of 10 and 5 (Fig. 9b). The light flux is transported thought the guides to the interior spaces being able to provide an optimal flux rate of light with minimal losses.

By software simulations, a solar collection system is placed to the top of the guide providing a centred light emitter of a semi-angle of $30^{\circ}$ at the input (0.01 area ratio). In addition, to show the size source influence in flux transmittance, an extensive light source has been used ( 1 area ratio). The influence of the light source area in transmission efficiency was determined from the light guide aspect ratio of 15 . Table 3 report the transmission efficiency collected at the output of the system of two prismatic guides for an aspect ratio of 15 . The perfect model and the prismatic guide with corner radius in peak prisms of $3 \mu \mathrm{m}$ which reproduce defects in prisms surface are compared. In the perfect prismatic guide, was obtained $98.79 \%$ of transmission efficiency. The bulk absortance influence is minimal, varying between $0.56 \%$ and $1.48 \%$. Thus, changes in light guide configurations provide a decreased use of prismatic material with consequent increase of available spaces. Optimizing the transmission

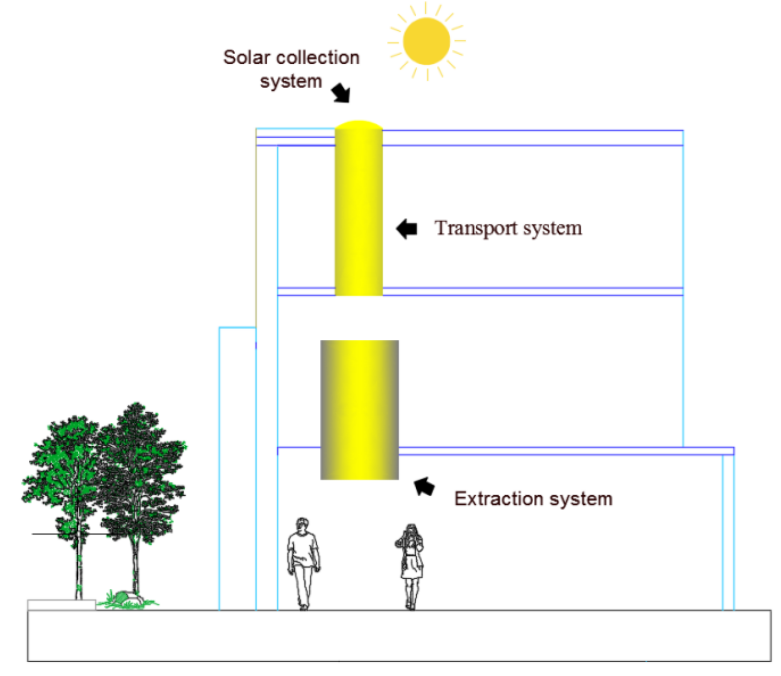

a)

b)



Figure 9: (a) Lighting system configuration based on CPLGs applied in a building and using the sunlight as a light source. (b) Raytracing simulations.

Table 3: Transmission efficiency in experimental assembly.

\begin{tabular}{lcc}
\hline \multicolumn{3}{c}{ Transmission efficiency $(\%)$} \\
\hline Extense source & Small source \\
\hline Perfect Lightguide system & 97.63 & 98.79 \\
Lightguide System (corner radius $3 \mu \mathrm{m})$ & 84.67 & 87.49 \\
\hline
\end{tabular}


efficiency ratio is also possible thanks to the improvements of the prismatic film structure and optimized guidance system designs.

\section{CONCLUSIONS}

An improved mathematical model is shown to predict light transmission efficiency in CPLGs based on realistic software simulations and experimental models. This model is based on semiempirical transmittance data and accurately predicts the influence of most important parameters such fabrication defects in prism corners, material absorptance, residual defects and the input light size dependence. The influence of the input light beam angle is studied to specify the angular acceptance range for optimal transmission of light.

\section{ACKNOWLEDGEMENTS}

The authors would like to thank Lambda Research Corporation for providing a university license for the ray tracing software.

\section{REFERENCES}

[1] Vázquez-Moliní, D., González-Montes, M., Fernández-Balbuena, A., García-Botella, A., Pohl, W., Galan, T. \& Bernabéu, E., Horizontal daylighting system for office buildings. Energy and Buildings, 67, pp. 525-530, 2013. http://dx.doi.org/10.1016/j.enbuild.2013.08.040

[2] García-Fernández, B., Vázquez-Molini, D. \& Fernández-Balbuena, A., Lighting quality for aluminum and prismatic light guides. In SPIE Optical Systems Design. International Society for Optics and Photonics, pp. 81700T-81700T, 2011.

[3] Whitehead, L.A., Dosanjh, P. \& Kan, P., High-efficiency prism light guides with confocal parabolic cross sections. Applied Optics, 37(22), pp. 5227-5233, 1998. http://dx.doi.org/10.1364/AO.37.005227

[4] Vázquez-Moliní, D., Álvarez Fernández-Balbuena, A. \& García-Fernández, B., Dielectric material. Natural Lighting Systems Based on Dielectric Prismatic Film, InTech, 2012.

[5] Whitehead, L.A., Nodwell, R.A. \& Curzon, F.L., New efficient light guide for interior illumination. Applied Optics, 21(15), pp. 2755-2757, 1982. http://dx.doi.org/10.1364/AO.21.002755

[6] Saxe, S.G., Prismatic film light guides: performance and recent developments. Solar Energy Materials, 19(1), pp. 95-109, 1989. http://dx.doi.org/10.1016/0165-1633(89)90026-9

[7] García-Fernández, B., Vázquez-Moliní, D., Fernández-Balbuena, A.Á., García-Botella, A. \& Antón, J.C.M., Light losses in hollow, prismatic light guides related to prism defects: a transmittance model. Chinese Optics Letters, 13(9), p. 092201, 2015. http://dx.doi.org/10.3788/COL201513.092201

[8] TracePro® Opto-Mechanical Design Software, Available at www.lambdares.com

[9] 2301 OLF Data sheet, Available at: http://multimedia.3m.com/mws/media/ 1132683O/3mtm-optical-lighting-film-2405.pdf?fn=OLF\%202405_Tech\%20Data_ Rev\%20B.pdf

[10] Whitehead, L.A., Simplified ray tracing in cylindrical systems. Applied Optics, 21(19), pp. 3536-3538, 1982. http://dx.doi.org/10.1364/AO.21.003536

[11] Nelder, J.A. \& Mead, R.A., Simplex method for function minimization. The Computer Journal, 7(4), pp. 308-313, 1965.

http://dx.doi.org/10.1093/comjnl/7.4.308 